\title{
THE IMMERSION DEPTH INFLUENCES ON CUTTING EDGE RADIUS DURING DRAG FINISHING
}

\author{
Ondřej Hronek, Miroslav Zetek \& Tomáš Bakša
}
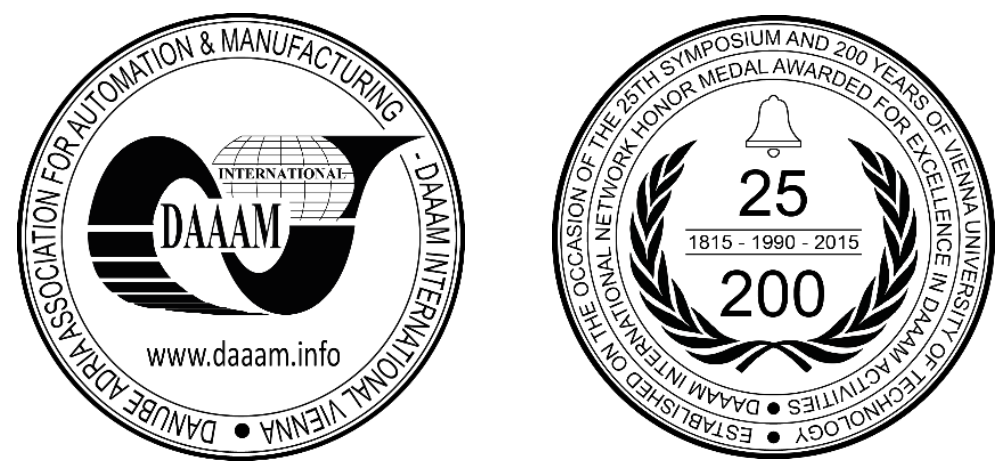

This Publication has to be referred as: Hronek, O[ndrej]; Zetek, M[iroslav] \& Baksa, T[omas] (2017). The Immersion Depth Influences on Cutting Edge Radius During Drag Finishing, Proceedings of the 28th DAAAM International Symposium, pp.1030-1036, B. Katalinic (Ed.), Published by DAAAM International, ISBN 978-3-902734-11-2, ISSN 1726-9679, Vienna, Austria

DOI: $10.2507 / 28$ th.daaam.proceedings.143

\begin{abstract}
The article deals with the intensity of the preparation of a cutting edge during the drag finishing process. The cutting edge radius of a milling tool is investigated because it is a very important element of cutting tool behaviour during machining. The intensity of the process was evaluated relative to the immersion depths of samples during drag finishing. The aim of this experiment is to confirm the idea that a greater depth in the working container causes a higher intensity of the process. A DF - 3 drag finishing machine was used for the experiment. An Alicona IFM G4 microscope was used to measure the microgeometry of the cutting edges. Cutting edges were always measured before and after the modification. The main goal of this article is to find the dependence between cutting edge modification intensity and immersion depth.
\end{abstract}

Keywords: Drag finishing; Process conditions; Immersion depth; Cutting edge modification

\section{Introduction}

Drag finishing is one of four types of mass finishing. Rotors and holders make the main rotary movements. There are multiple combinations of rotary motions. The samples are attached in the holders for finishing. The holder and rotors can make counter-clockwise and clockwise motions. The holders also make a vertical movement in the working container. The container does not move. High circumferential speeds and great depth of immersion cause high pressure between the media and the parts. [1] [2]

In mechanical engineering this method is mainly used for finishing milling and drilling tools. It is especially used in the cutting edge preparation of cutting tools. Cutting edges do not have a high quality after grinding. [3] Defects can be seen on the cutting edge under a microscope. For example, the flank and rake surfaces are badly shaped, there are small areas on the main edges where the cutting edge is missing, or there is a variable size of the radius. Most defects on the cutting edges are removed by drag finishing, which creates a higher quality cutting edge. The cutting edge has a better surface roughness and a modified microgeometry with a precise value of the radius. [4] Also the cutting edge radius affects cutting tool wear. [8] The influence of cutting edge microgeometry was proved when milling super-alloy Inconel 718 during a practical experiment with different cutting edge radii $(15 \mu \mathrm{m} ; 20 \mu \mathrm{m} ; 25 \mu \mathrm{m})$. [9] 
In many cases other experiments are performed after drag finishing. These experiments monitor the cutting tools in the process of machining. [5] [6] Drag finishing is a common process prior to the deposition of thin coatings on cutting tools. This type of machining improves the adhesive properties of the tools. This technology can create a barrier on the surface which prevents the development of cracks or other defects, so this is a big advantage. Different radius sizes cause changes to the effect and the size of the cutting forces. In many cases, drag finishing causes longer tool life. [7]

\subsection{Problem statement}

Cutting tool microgeometry is a topical issue. As microgeometry affects cutting tool life and efficiency, research and development need to continue and look for new possibilities for improving cutting tool quality. Therefore, this article is concerned with cutting tool microgeometry modification. The effects of the drag finishing process parameters are investigated on the cutting edge radius. The cutting edge radius is one of the most important parameters on a cutting tool. This article investigates the effect of the immersion depth on the cutting edge radius. We investigated how the immersion depth affects the intensity of the microgeometry modification. The research is also focused on the positive or negative impact of immersion depth on the shape and quality of the cutting tool wedge microgeometry.

\section{The apparatus used in the experiment}

The IFM G4 microscope is a 3D optical / scanning measuring device which works on the principle of changing focal lengths. It is used for measuring surface characteristics, analysis and also for actual dimensions. The accuracy of the device is in nanometers. It is also suitable for cutting edge measurement.

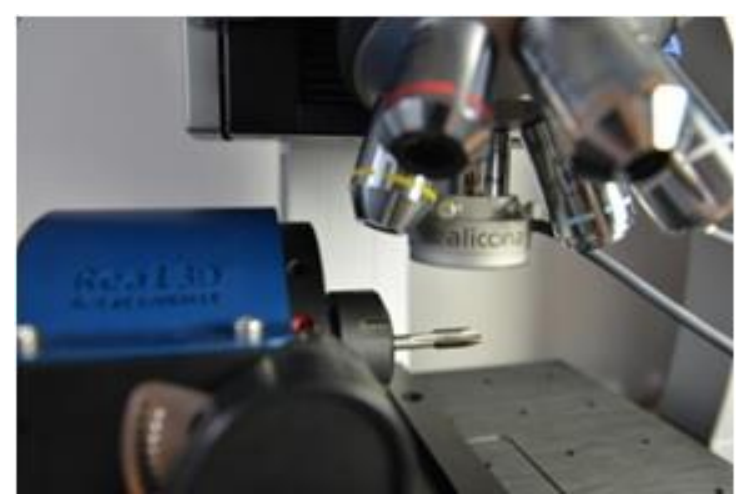

Fig. 1. IFM G4 Microscope

A DF - 3 drag finishing machine is used for preparing the edges of the tools for machining. The main movement is a planetary rotary motion made by the holders and the rotors. This movement causes pressure between the media and the workpieces. 16 workpieces can be clamped into the DF - 3 at the same time. A big advantage is the simplicity of the drag finishing machine. The drag finishing machine has five main parts: the frame, the control panel, the process container, the rotor and the holders.

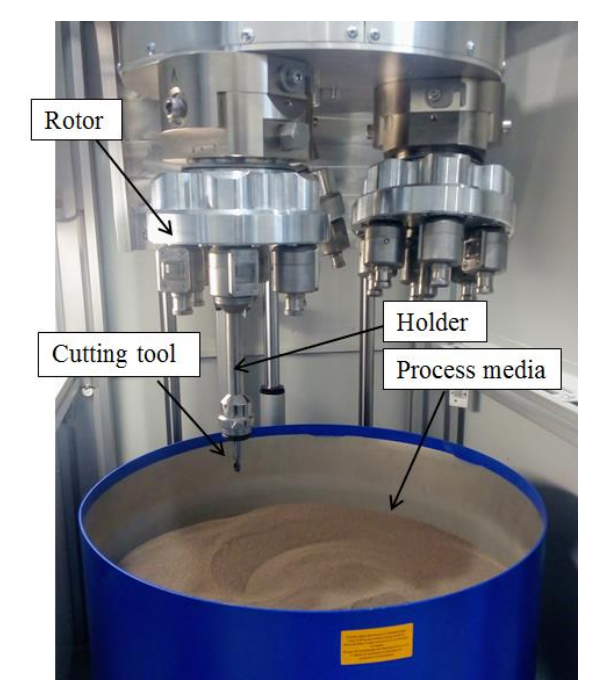

Fig. 2. Drag finishing machine 
HSC 1/300 medium was used for experiment. The medium consists of two components: walnut shells and silicon carbide. It is a dry type of abrasive. It is used only for drag and stream finishing. HSC 1/300 is used mostly for finishing cutting tool microgeometry. For an efficient drag finishing process with a short time by using HSC 1/300, the maximum value of the cutting edge radius is $20 \mu \mathrm{m}$.

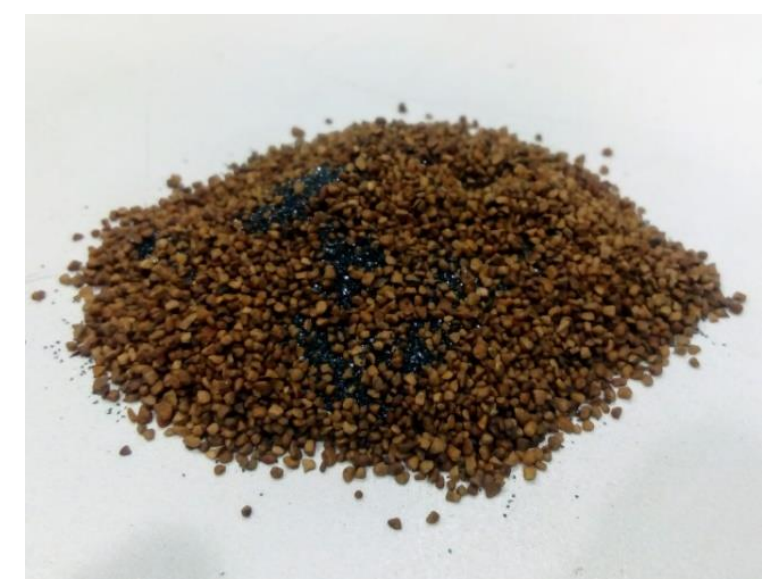

Fig. 3. Process media HSC 1/300

\section{Experiment}

The tool diameter is $8 \mathrm{~mm}$ and the length is $55 \mathrm{~mm}$. Sintered carbide milling tools were used for the experiment and evaluating the cutting tool microgeometry. Twelve end mill tools were used and six tests were prepared. There were two samples for different immersion depths (Table 1.).

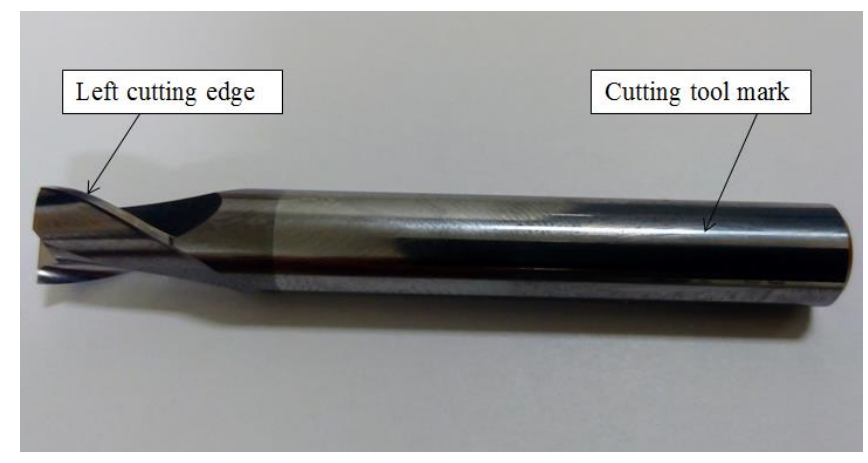

Fig. 4. Modified cutting tool

Before the experiment, it was necessary to select the appropriate finishing parameters. The DF -3 drag finishing machine has six parameter settings. The aim is to find out the relationship between the intensity and the immersion depth of drag finishing. The Table 1. shows that the only variable parameter is the immersion depth. Other parameters are constant during the experiment.

\begin{tabular}{|c|c|c|c|c|c|}
\hline $\begin{array}{c}\text { Overall time } \\
{[\mathbf{m i n}]}\end{array}$ & $\begin{array}{c}\text { CCW } \\
{[\mathbf{m i n}]}\end{array}$ & $\begin{array}{c}\text { CW } \\
{[\mathbf{m i n}]}\end{array}$ & $\begin{array}{c}\text { Rotor speed } \\
{\left[\mathbf{m i n}^{-1}\right]}\end{array}$ & $\begin{array}{c}\text { Holder speed } \\
{\left[\mathbf{m i n}^{-1}\right]}\end{array}$ & $\begin{array}{c}\text { Immersion } \\
\text { depth [mm] }\end{array}$ \\
\hline 6 & 3 & 3 & $40 /-40$ & $65 /-65$ & 330 \\
\hline 6 & 3 & 3 & $40 /-40$ & $65 /-65$ & 350 \\
\hline 6 & 3 & 3 & $40 /-40$ & $65 /-65$ & 370 \\
\hline 6 & 3 & 3 & $40 /-40$ & $65 /-65$ & 390 \\
\hline 6 & 3 & 3 & $40 /-40$ & $65 /-65$ & 410 \\
\hline 6 & 3 & 3 & $40 /-40$ & $65 /-65$ & 430 \\
\hline
\end{tabular}

Table 1. Process parameters for drag finishing 
All 12 samples were measured by IFM before finishing. The most important parameter was the radius of the cutting edge. The left and right edges were measured separately. The scanning area was $2 \mathrm{~mm}$ from the tip of the milling tool.

\begin{tabular}{|c|c|c|}
\hline Sample number & $\begin{array}{c}\text { Left radius edge } \\
{[\boldsymbol{\mu m}]}\end{array}$ & $\begin{array}{c}\text { Right radius edge } \\
{[\boldsymbol{\mu} \mathbf{m}]}\end{array}$ \\
\hline 1 & 1.31 & 1.73 \\
\hline 2 & 1.55 & 1.39 \\
\hline 3 & 1.66 & 1.27 \\
\hline 4 & 1.33 & 1.61 \\
\hline 5 & 1.26 & 1.83 \\
\hline 6 & 1.66 & 1.23 \\
\hline 7 & 1.47 & 1.51 \\
\hline 8 & 1.30 & 1.36 \\
\hline 9 & 1.90 & 1.39 \\
\hline 10 & 1.24 & 1.26 \\
\hline 11 & 1.66 & 1.54 \\
\hline 12 & 1.48 & 1.36 \\
\hline
\end{tabular}

Table 2. Values of cutting edge radius before drag finishing

Fig. 5. shows the cutting edge of the sample. This edge was created by grinding. It is magnified 20x by an Alicona IFM microscope. At this magnification it is easy to identify the marks created by the grindstone. Moreover, the radius of the cutting edge is also evident, even though it is only about 1.47 microns. Despite the fact, that the cutting tool is much sharper after grinding, the cutting edge is brittle and prone to chipping and also to deformation.

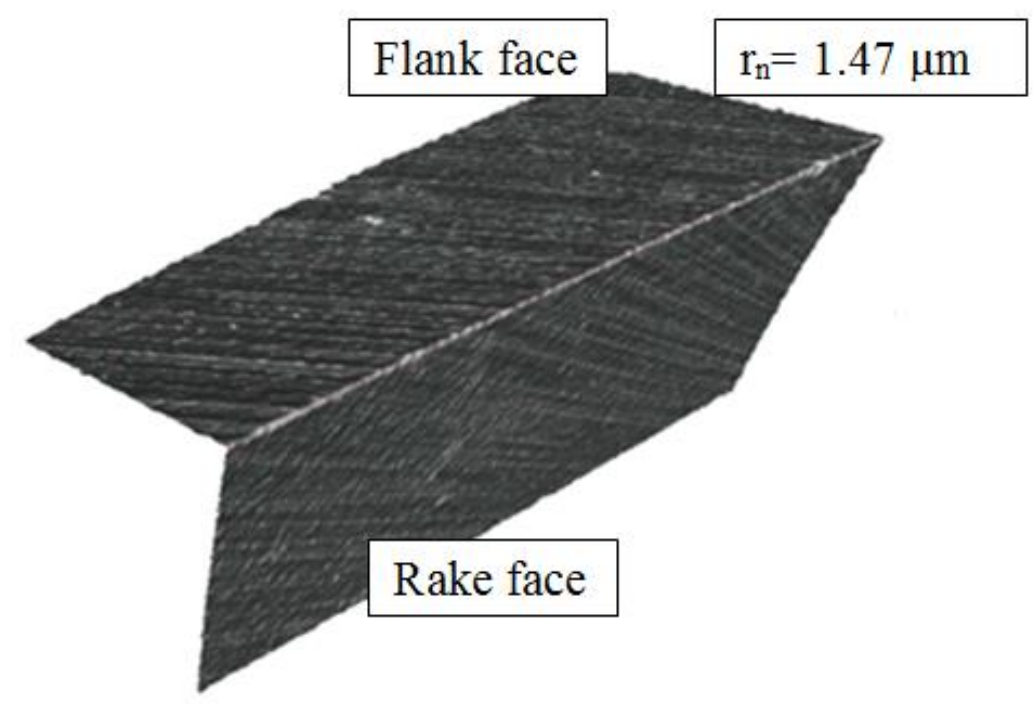

Fig. 5. Cutting edge microgeometry before drag finishing

\section{Results}

Twelve samples were finished at six different depths. The depth was changed after every second process. The immersion depth increased in $20 \mathrm{~mm}$ intervals. The difference between the initial and the final depth was $100 \mathrm{~mm}$. Every cutting edge was measured by IFM after drag finishing. The left and right radii of the cutting edge were measured at the same location as before drag finishing. The measuring point was on the edge, $2 \mathrm{~mm}$ from the tip (Fig. 6.). 


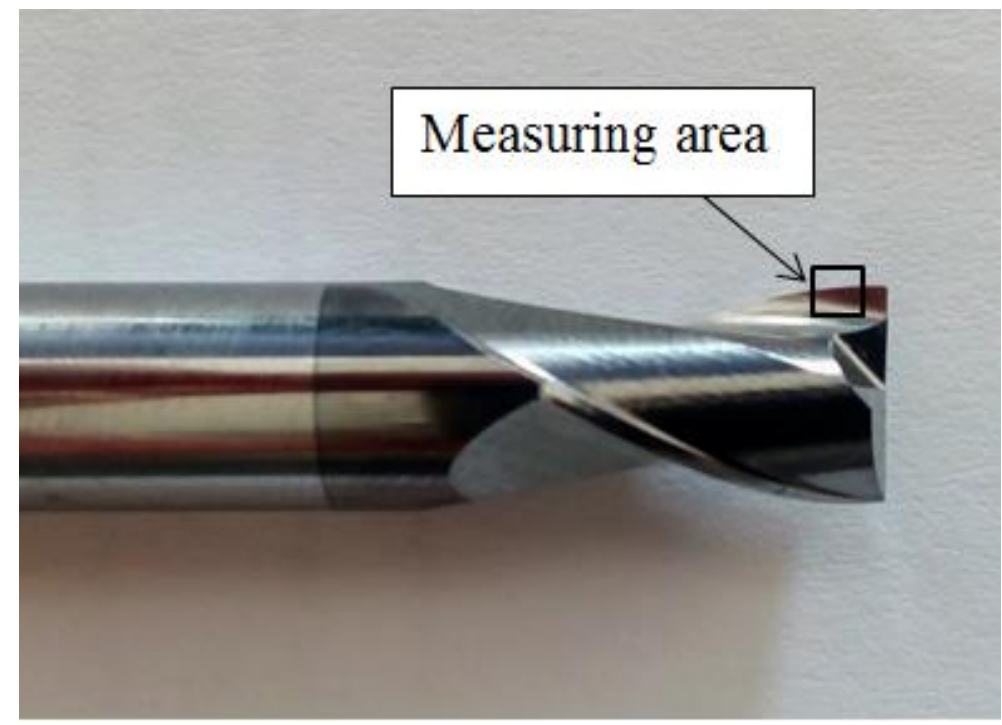

Fig. 6. Example of measuring area on cutting edge

The smallest measured radius was 8.1 microns on the sample with $330 \mathrm{~mm}$ immersion. The biggest radius was achieved at the bottom of the process container $(430 \mathrm{~mm})$. This was 14.6 microns. So, with a distance of $100 \mathrm{~mm}$, the intensity of finishing the radius increases by 6.5 microns. At an immersion depth of $430 \mathrm{~mm}$, the radius of the sample was $44 \%$ larger than the sample at a depth of $330 \mathrm{~mm}$ with the same entry condition. Fig. 7 . shows the increase in the size of the radius by drag finishing. The values of the radius increase gradually. The biggest increase of the intensity is between depths of 390 and $410 \mathrm{~mm}$. The increase of the cutting edge was more than 2 microns at this point. The curves on the graph have relatively the same shape. The radius sizes for the left and right milling edges are approximately the same. The biggest difference was measured at a depth of $410 \mathrm{~mm}$. The difference between the left and right cutting edges was 0.74 microns.

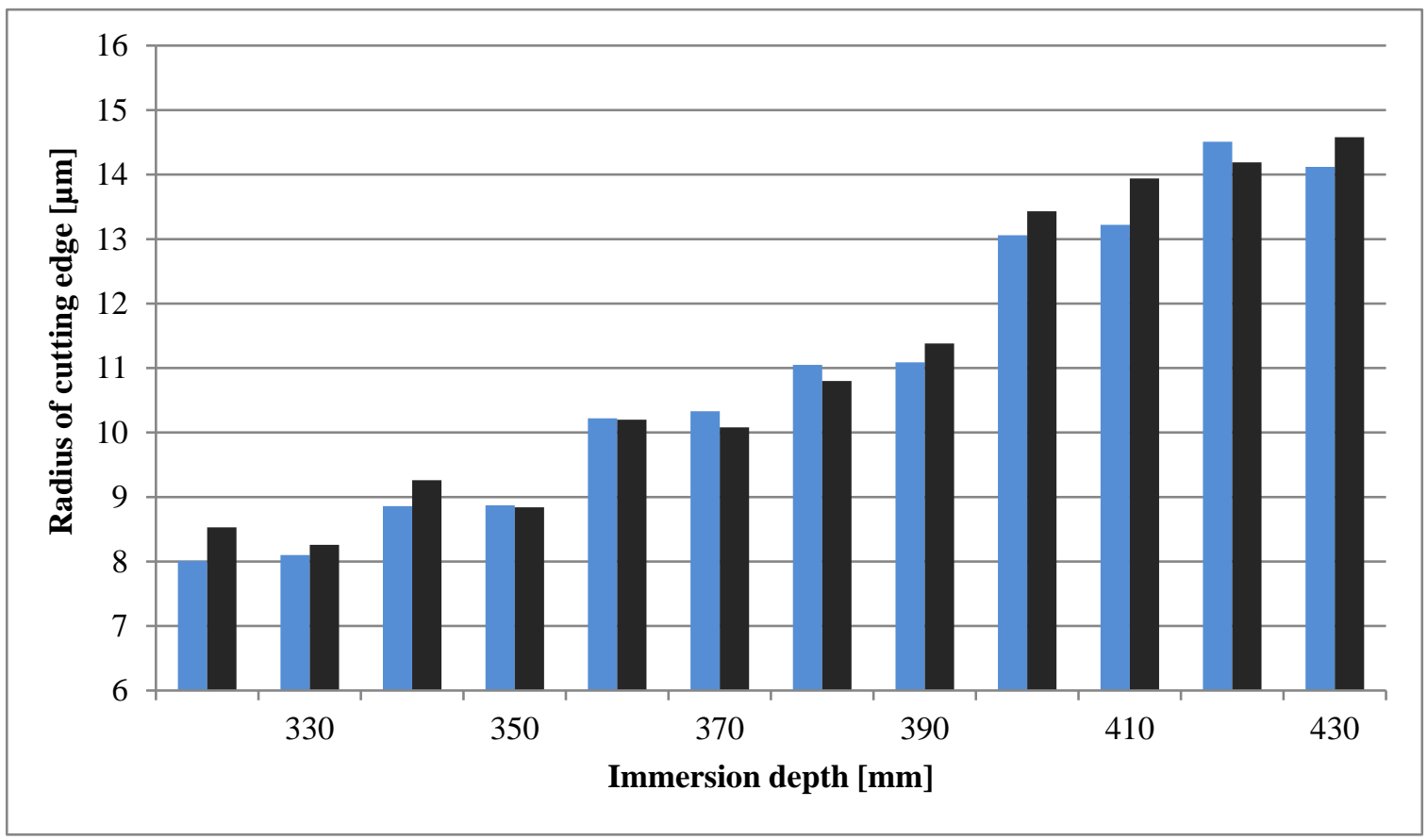

Fig. 7. Results of experiment

Fig. 8. shows the development of the cutting edge before and after finishing at depths of $330 \mathrm{~mm}$ and $430 \mathrm{~mm}$. There is a gradual enlarging of the cutting edge radius. There is also a difference in the flank and rake surfaces. The quality of these surfaces improved with greater depth. 


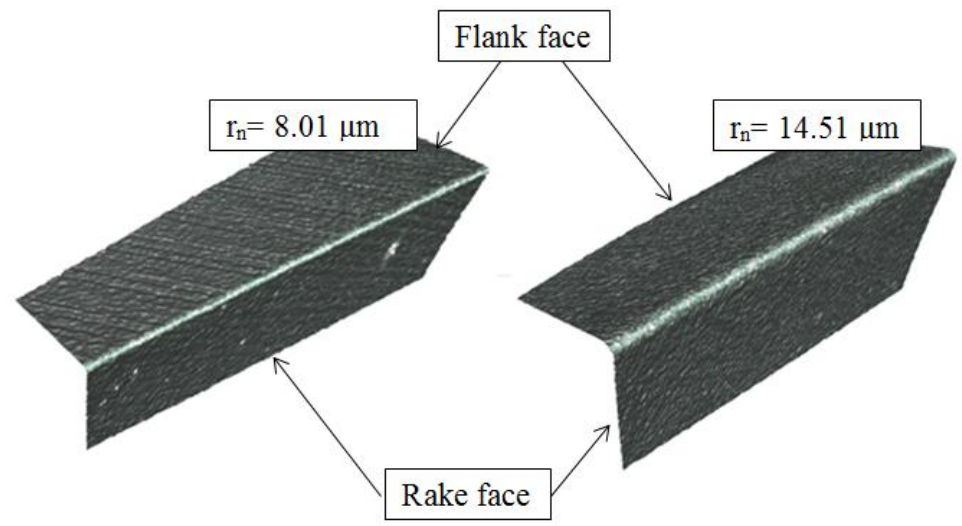

Fig. 8. Cutting edge microgeometry after modification; immersion depth $330 \mathrm{~mm}$ and $430 \mathrm{~mm}$

Differential analysis was used to make an interesting comparison between the cutting edge before and after finishing. Differential analysis is based on the color spectrum and provides the user with quick and clear information. Fig. 9. shows the edge which was finished at $330 \mathrm{~mm}$. The radius of the cutting edge is 8.1 microns.

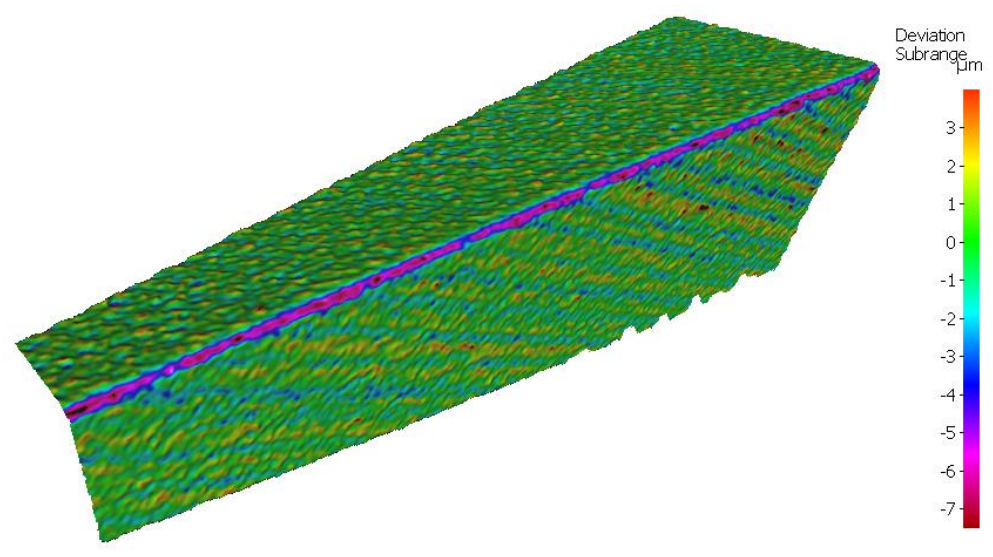

Fig. 9. Differential analysis; immersion depth $330 \mathrm{~mm}$

The cutting edge in Fig. 10. is finished at an immersion depth of $430 \mathrm{~mm}$. The radius of the edge is 14.1 microns. Compared with Fig. 9., it is evident that the radius has increased. Additionally, the finishing at $430 \mathrm{~mm}$ gives better surface quality, because there are fewer tracks from the grindstone.

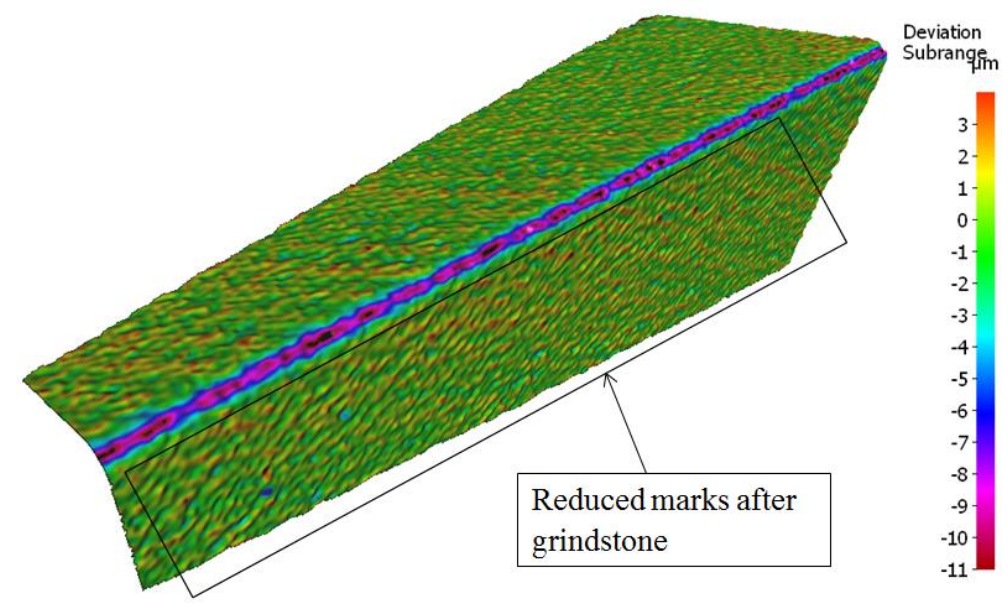

Fig. 10. Cutting edge microgeometry; immersion depth $430 \mathrm{~mm}$ 


\section{Conclusion}

The main focus of this experiment was to verify the influences of the immersion depth on the cutting edge radius of an end mill tool. Therefore, an experiment was proposed in which the drag finishing immersion depth was changed. Walnut shells with $\mathrm{SiC}$ were used as the medium for the experiment. Immersion depths of $330 \mathrm{~mm} ; 350 \mathrm{~mm} ; 370 \mathrm{~mm}$; $390 \mathrm{~mm} ; 410 \mathrm{~mm}$ and $430 \mathrm{~mm}$ were selected. By evaluating the measured values, the relationship was found between the cutting edge radius and the immersion depth during the drag finishing process. The smallest radius, 8.1 microns, was measured at a depth of $330 \mathrm{~mm}$, whereas at the depth of $430 \mathrm{~mm}$, the value of the radius increased by $44 \%$, to 14.6 microns.

Besides from the fact, that a deeper immersion depth causes a higher intensity of cutting edge modification, marks after grindstone disappear from flank and rake face. Another advantage is that the symmetry of cutting edge is achieved after drag finishing. Functional areas of the cutting tool with higher qualities are necessary not just for cutting process, but also for a coating process. It exists many cases, where cutting edge modification has brought a cutting tool life increasing, for example during machining stainless steel. But it is important to realize, that materials with toughness, such as $\mathrm{Al}$ and $\mathrm{Cu}$ alloys can't be machined by tools with high value of cutting edge radius. So it is necessary to understand a system, cutting tool - microgeometry - cutting material - workpiece material for proper cutting edge modification.

In the near future, these tools will be tested. This is necessary in order to obtain relevant information about the potential influence of microgeometry on the durability of the cutting tool. At the same time, cutting forces will be measured during the experiment. This will provide additional information and the relationships between the cutting tool microgeometry and the cutting process. This article is one of the tests that are designed to increase the quality of monolithic carbide end mill cutters.

\section{Acknowledgments}

This paper includes results created within the project SGS-2016-005.

\section{References}

[1] INFOCUBE.CZ [online]. [Accessed: 2017-09-15]. http://infocube.cz/wp-content/uploads/2017/04/Vlečné-omílánízlepšuje-kvalitu-nástrojů.

[2] Pospíšilová, I., Vojtěch, D. \& Kubásek, J. (2014). Structure and Properties of Zn-Mg Alloys for Medical Implants. Manufacturing Technology, Vol. 14, No. 3, pp. 422 - 427, ISSN: 1213 - 2489

[3] Čilliková, M., Mičúch, M., Neslušan, M. \& Mičietová, A. (2013). Non-destructive micromagnetic evaluation of surface damage after grinding. Manufacturing Technology, Vol. 13, No. 2, pp. 152 - 157, ISSN: 1213 - 2489

[4] Mádl, J., Razek, V., Koutný, V. \& Kafka, J. (2013). Surface Integrity in Notches Machining. Manufacturing Technology, Vol. 13, No. 2, pp. 188 - 193, ISSN: 1213 - 2489

[5] Mádl, J. \& Martinovský, M. (2015). Cutting Tool Wear Monitoring. Manufacturing Technology, Vol. 15, No. 3, pp. 380-384, ISSN: $1213-2489$

[6] Česáková, I., Zetek, M. \& Švarc, V. (2013). Evaluation of Cutting Tool Parameters, Proceedings of the 24th DAAAM International Symposium, pp. 1105 - 1114, Published by Elsevier, ISBN 978-3-902734-07-5, ISSN 18777058, Zadar, Croatia

[7] Pilc, J. \& Vasilko, K. (2013). Development and applications of a rotating turning tool. Manufacturing Technology, Vol. 13, No. 2, pp, 226-231, ISSN: 1213 - 2489.

[8] Lenz, J. \& Westkaemper, E. (2017). Wear Prediction of Woodworking Cutting Tools based on History Data. Procedia CIRP, Vol.63, pp 675 - 679, ISSN: 2212 - 8271

[9] Hronek, O. (2017). The Influence of the Cutting Tool Surface Quality on Durability, Diploma thesis, Department of Machining Technology, University of West Bohemia, Pilsen, Czech Republic

[10] Baksa, T., Schornik, V., Adamek, P. \& Zetek, M. (2016). Machining of Inconel 718 Using Uncoated Cutting Tools with Different Cutting Edge Quality, Proccedings of the 27th DAAAM International Symposium, pp. 0441 - 0446, B. Katalinic (Ed.), Published by DAAAM International, ISBN 978-3-902734-08-2, ISSN 1726-9679, Vienna, Austria 